\title{
Investigation of changing pressure and drag coefficient on square cylinder with different corner in air flow
}

\author{
Ali $\operatorname{Kargar}^{1}$, Majid Lotfi ${ }^{2}$
}

\begin{abstract}
In this research, numerical analysis of corners of square cylinder on following parameters of the turbulence and changing pressure and drag coefficient in air flow has been discussed. Selected models were made of Plexiglas that side length is $15 \mathrm{~mm}$ and high of this is $400 \mathrm{~mm}$. To investigate effect of different corners on following parameters of square cylinder, these cylinders with sharp, rounded and beveled corners are used. Amount of bevel and radius of rounded edges are selected 25.5mm. Type of grid (mesh) around each square is structured and for other parts of solution range, unstructured mesh was used. The result of this research, shows angle of attack and figure of corners of cylinder, causes significant changes in turbulence parameters of square cylinder and value of drag coefficient. Rounding and bevel corners of square cylinder, decrease mean (average) and maximum turbulence in the wakes of cylinder. Drag coefficient by rounding and beveling corners of square cylinder with sharp edges can be considerably reduced.
\end{abstract}

Keywords - aerodynamics, wakes of square cylinder, corners of the cylinder, drag coefficient

\section{Introduction}

Compressible flow with friction around the cylinder is the classic problems of mzechanic of fluids. Despite the simple geometry of the cylinder, the flow of back is very complex and sophisticated. Especially in high Reynolds, momentum equations from analytical methods are not easily obtained. Therefore (for this reason) numerical analysis of the wake behind the cylinder is a fundamental and dynamic issue in mechanic of fluids.

Several factors are influencing the feature of wake of square cylinder that such as effect of Reynolds number, blockage ratio, ratio of view, surface roughness, dimension of terminal holder plates cylinder, turbulence of input stream (inlet flow), the edges of the cylinder and the angle of attack. In this study, the effect of corners of square cylinder on turbulence parameters in the intensity of input turbulence with rate of 0.1 percent has been investigated. Review of previous works: many experimental and numerical studies on the flow around a cylinder with a square cross-section were performed and this subject is major and interesting issue for researchers.

Sohankar and et all [1] in 1995, in their study, investigate numerical flow around a square cylinder for Reynolds number 2200 and the results were compared with

1- $\quad$ Ali Kargar (corresponding author)

Department of Mechanical Engineering, Quchan Branch, Islamic Azad University, Quchan, Iran

\section{2- $\quad$ Majid Lotfi}

Department of Chemical Engineering, Quchan Branch, Islamic Azad University, Quchan, Iran experimental values and this result were satisfactory. Lee [2], in his study, investigates the effect of rotation square cylinder on mean and oscillatory pressure field at Reynolds number 180000. Hasan [3], examined experimental studies on flow around square cylinder at different angles and Reynolds number 6700 up to 43000 .

Sukhtanlou and et all [4], in their study, investigate experimental flow around a square cylinder at different angles of attack using hot-wire speedometer. Their result show, angle of attack had a remarkable effect on the value of drag coefficient of square cylinder. Tamura and Miyagi [5], in their study, investigate experimental two and three dimensional flow around square cylinder with a different angles of attack and Reynolds number 30000. They also, investigate drag and lift coefficient cylinder in different intensity of turbulence of input stream on cylinder and different forms of corners of square cylinder. They, in their research achieved mean (average) velocity profiles and intensity of turbulences and values of drag coefficient at zero degrees angle of attack and Reynolds number 13, 200 around square cylinder with side length $15 \mathrm{~cm}$. Tamura and Miyagi [6] also in that year, investigate, numerical effect of form the corners of square cylinder on pressure distribution. Their result of research that considered three dimensional and compressible flow, show, and changing form in edges of square cylinder that causes decrease in lift and drag coefficient, and number will increase. In 1988, Kawai [7], in his study, investigate the empirical effects of corners on aero elastic instabilities of high buildings.

\section{Physical Model}

Length of cylinders used in these experiments is $40 \mathrm{~cm}$ and side length is $15 \mathrm{~mm}$. Type of three models is selected Plexiglas and thus, effect of surface roughness doesn't appear in the results. Corners of the model have been selected in Fig 1. One of models has sharp edges and in two other model edge of cylinder rounded and bevelled in $2.5 \mathrm{~mm}$. Bevelling edges of cylinder did by milling operation and rounding edges of cylinder by turnery operation.

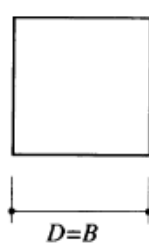

sharp corners

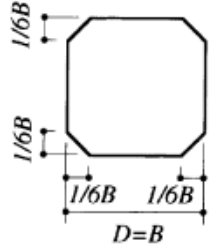

chamfered corners

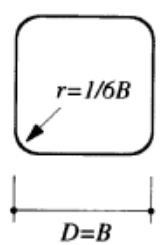

rounded corners
Figure 1. The cylinders with different corners 


\section{The Governing Equations}

Numerical analysis of air flow on cylinder is done by applying continuity and momentum rules in a very small element and integrating from results on the entire range. The governing equation for determining distribution of pressure and velocity on cylinder can be expressed as follows:

$$
\begin{gathered}
\frac{\partial \rho}{\partial t}+\nabla \cdot(\rho V)=0 \\
\rho \frac{D V}{D t}=-\nabla P+(\nabla \cdot \tau)+\rho g
\end{gathered}
$$

Where $\rho$ fluid density and $\mathrm{V}$ is fluid velocity vector, $\rho D V / D t$ is mass accumulation, $\nabla P$ is pressure force, $\nabla . \tau$ is force of fluid viscosity and $\rho g$ is the gravitational force per unit volume of fluid. Due to the geometry of problem, the equations must be solved in cylindrical coordinates.

\section{Iv. Numerical Model}

Numerical analysis of equations in cylindrical coordinate and in three dimensional is difficult. At first, due to the symmetry condition and taking some appropriate and reasonable assumptions, we model the solution of flow in cylinder in two dimensional conditions. Numerical models in terms of taking relationship between pressure and velocity with different methods can be analyzed. In the constant velocity method $[8,9]$, with respect to the relationship between pressure and velocity, velocity is assumed to be constant and pressure is according to the velocity $[10,11]$. However in models with constant and variable static pressure, velocity with respect to the rate of constant selective pressure obtained. The simple algorithm for solving this problem is used.

\section{v. Mesh the Geometry}

In order to simulation, range of solution should be considered large. Thus, the behind the cylinder about 25 times, front of cylinder 15 times and upper and lower parts are considered 10 times larger that is shown in Fig 2. Type of mesh around square is structured and other parts of solving range are unstructured.

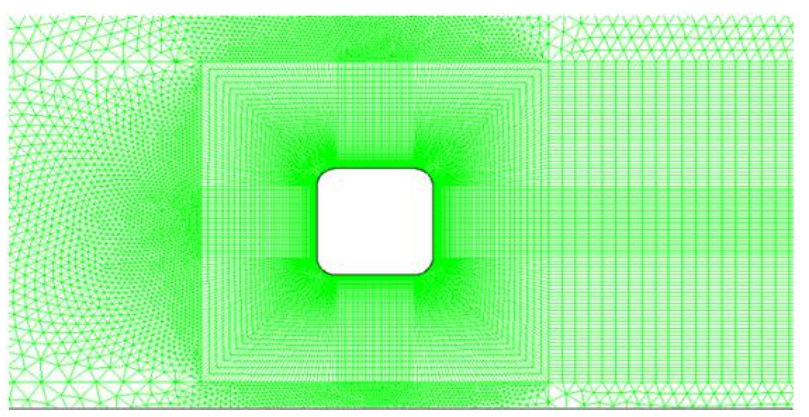

Figure 2. mesh the square cylinder

Around the cylinder has far field boundary condition with air velocity of $10 \mathrm{~m} / \mathrm{s}$. also the condition of surface of the cylinder should be considered wall.

\section{vi. Results}

In this section, diagrams of the velocity contours, lines of flow, drag coefficient, pressure coefficient and other contours for three types of square cylinder, are presented. In Fig 3 to 7, the velocity of contours, stream line, drag and pressure coefficient for square cylinder with sharp corners is shown:

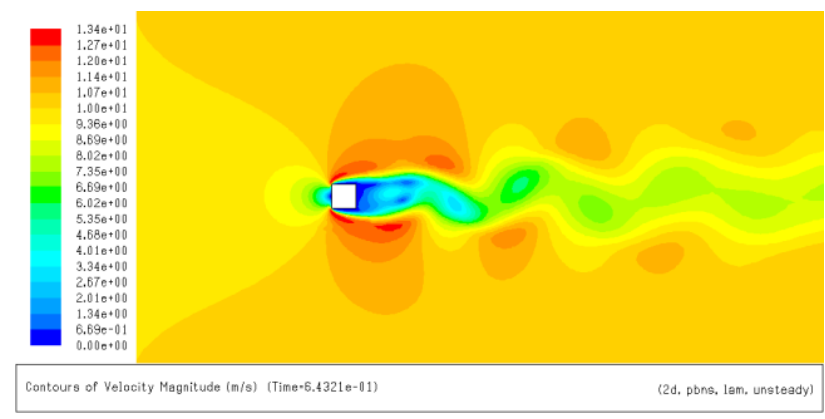

Figure 3. Velocity contours and display of vortices in sharp square cylinder

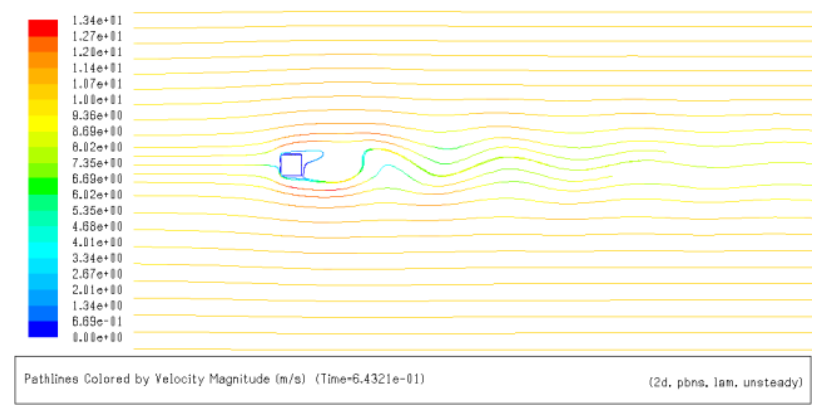

Figure 4. Velocity magnitude and display of vortices behind a square cylinder

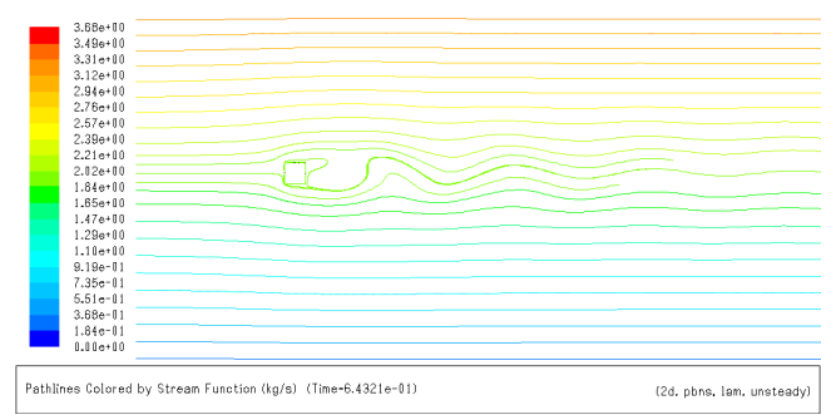

Figure 5. Stream function and display of vortices of sharp square cylinder

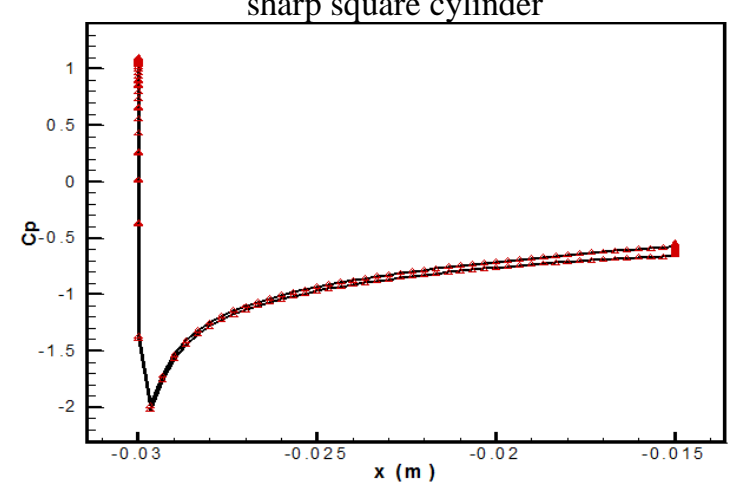

Figure 6. Variation of pressure coefficient on a sharp square cylinder 


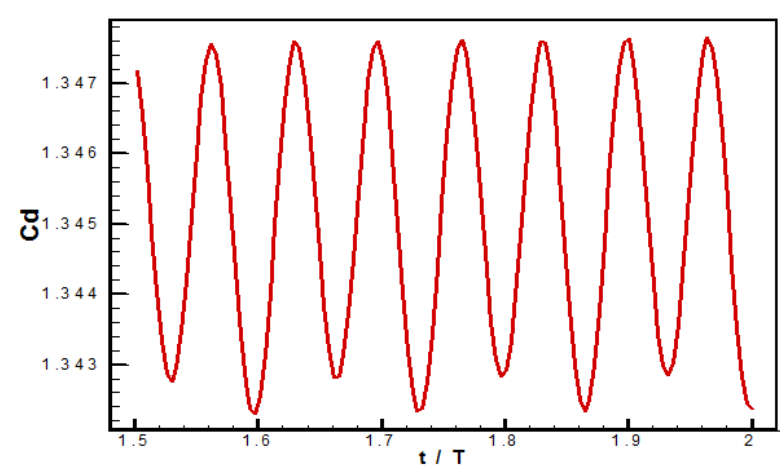

Figure 7. Variation of drag coefficient on a sharp square cylinder

In Fig 8 to 11, the velocity of contours, stream line and pressure coefficient for square cylinder with beveled corners is shown:

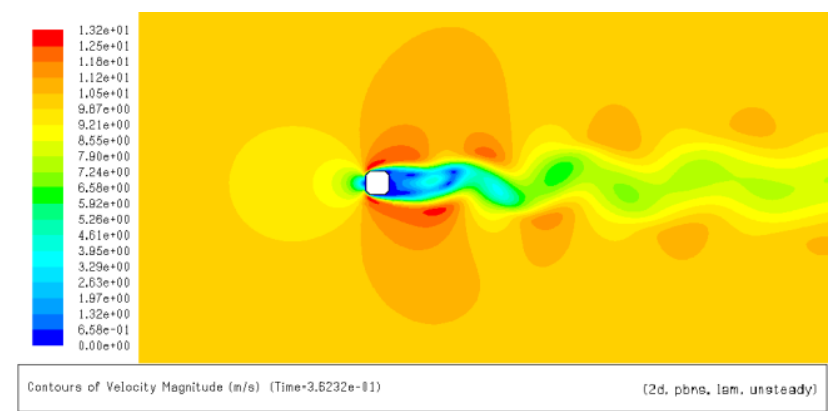

Figure 8. Velocity contours and display of vortices on sharp beveled cylinder

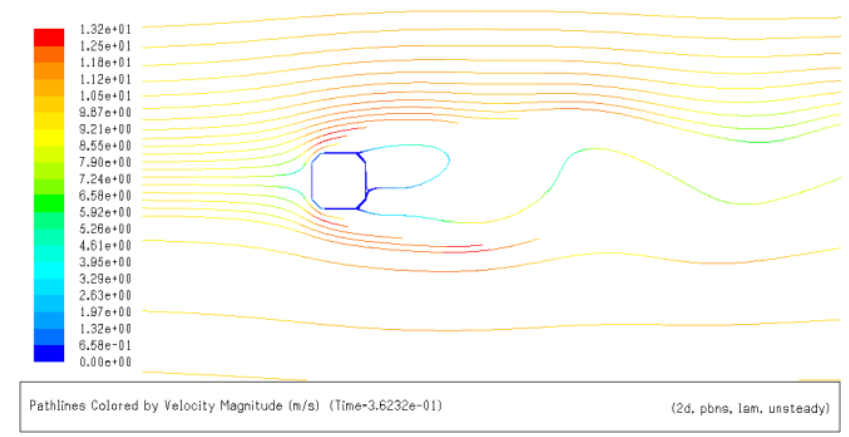

Figure 9. Velocity magnitudes and display of vortices behind a beveled cylinder

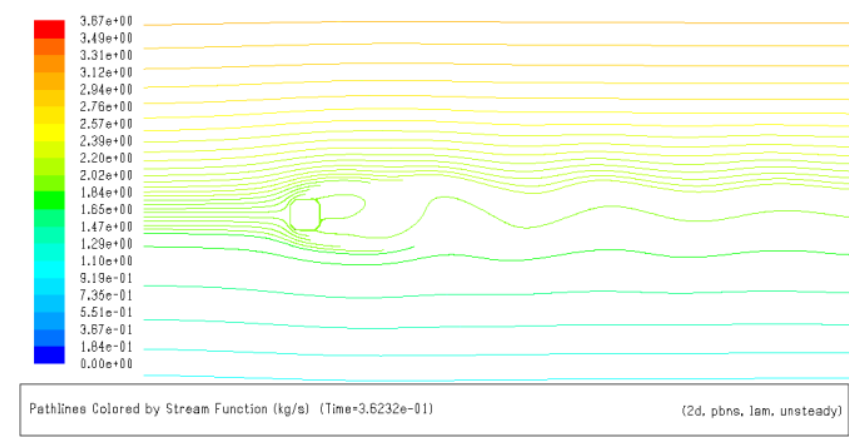

Figure 10. Stream function and display of vortices on sharp beveled cylinder

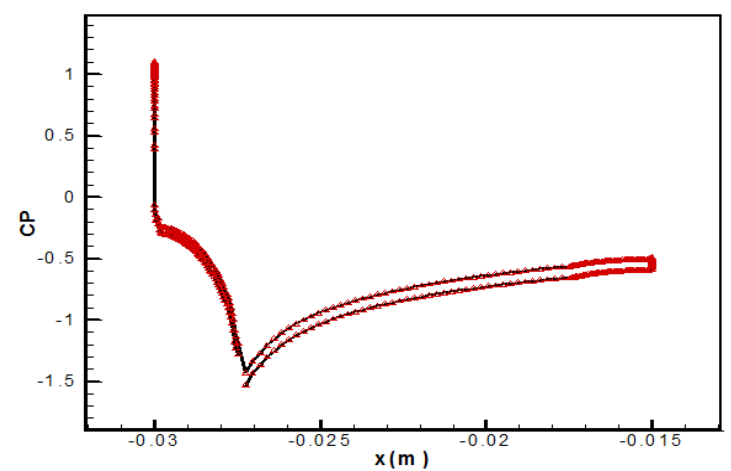

Figure 11. Variation of pressure coefficient on a sharp beveled cylinder

In Fig 12 to 15, the velocity of contours, stream line and pressure coefficient for square cylinder with beveled corners is shown:

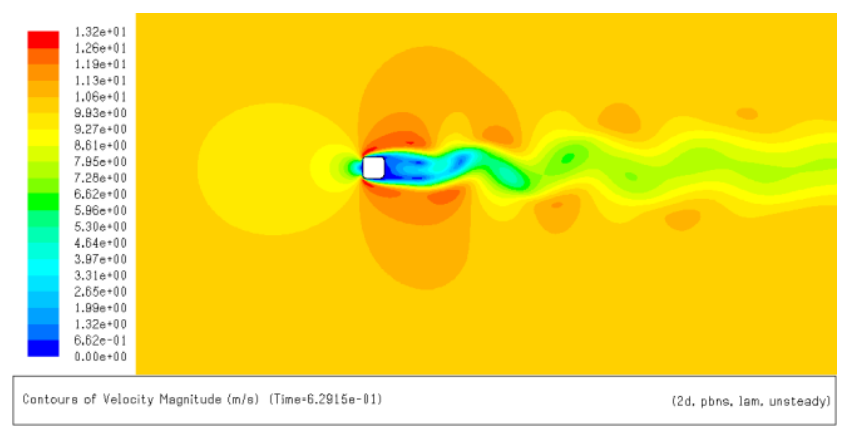

Figure 12. Velocity contours and display of vortices on sharp round cylinder

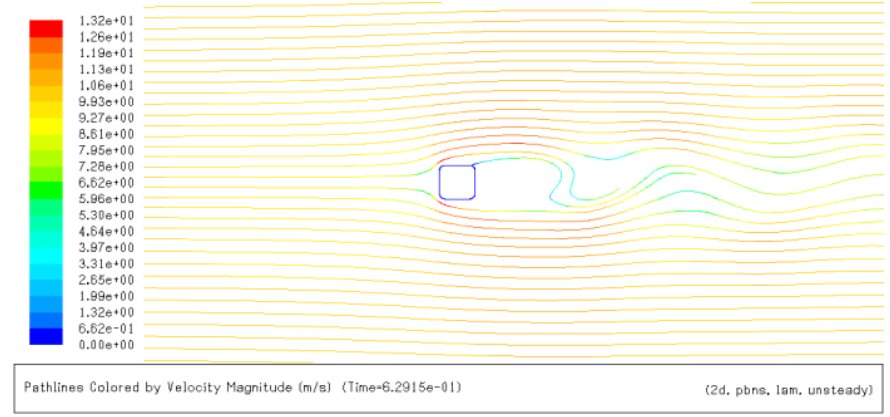

Figure 13. Velocity magnitudes and display of vortices behind a round cylinder

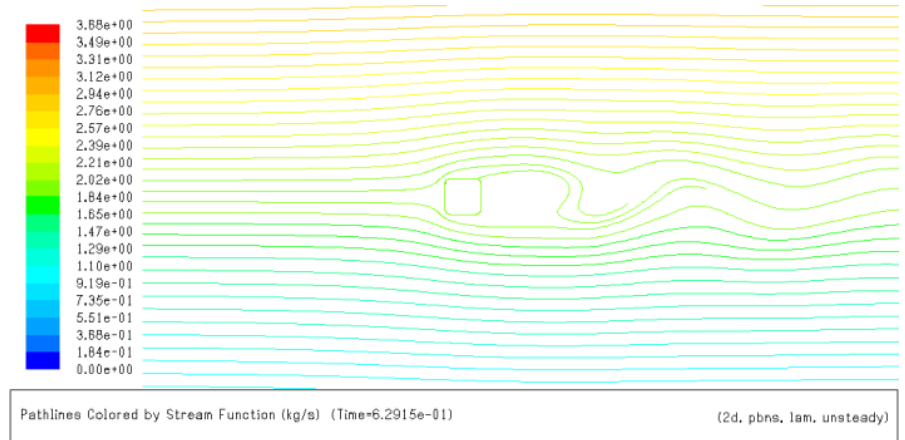

Figure 14. Stream function and display of vortices on sharp round cylinder 


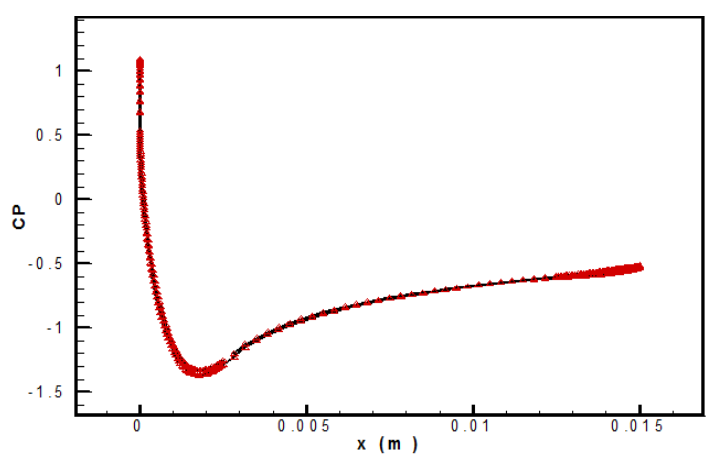

Figure 15. Variation of pressure coefficient on a sharp round cylinder

In table I, the mean values of drag coefficient for all three type of cylinder is given.

TABLE I. MEAN VALUES OF DRAG COEFFICIENT FOR ALL THREE TYPE OF CYLINDER

\begin{tabular}{|c|c|}
\hline $\begin{array}{c}\text { The corner of the square } \\
\text { cylinder }\end{array}$ & $\begin{array}{c}\text { THE MEAN OF DRAG } \\
\text { COEFFICIENT }\end{array}$ \\
\hline Sharp & 1.3425 \\
\hline Beveled & 1.1315 \\
\hline Round & 1.1122 \\
\hline
\end{tabular}

\section{vII. Conclusion}

At the present work, numerical analysis of the corners of the square cylinder on turbulence parameters on the wake changes in drag and pressure coefficient has been discussed.

- The value of mean and maximum turbulence intensity behind the square cylinder depends to the amount of distance from cylinder, attack angle and form of corners.

- Chamfering and rounding the corners of square cylinder, generally, leads to decrease in maximum and mean turbulence intensity in cylinder sequence.

- Along with chamfering and rounding the corners form of square cylinder with sharp corner, drag coefficient will notably decrease.

\section{References}

[1] sohankar A. Norberg C. Davidson L., 1995, A Numerical Study of unsteady two dimensional flow around rectangular cylinder at incidence, phd thesis, Chalmers University of Technology, Gotenberg, Sweden.

[2] Lee B.E, 1975, the effect of turbulence on the surface pressure field of a square prism, J.Fluid Mech, 69, 263-282.

[3] Hasan M.A.Z., 1989, the near wake structure of a square cylinder, Int. J. Fluid Flow, 10, 339-348.

[4] E. shokhtanlo, A. khoshnevis, 1998, Experimental investigation of corners` form influence on the turbulence parameters sequence of square cylinder, The 8th International Conference of Iranian Aerospace Society, Isfahan, Shahin Shahr, Malek Ashtar University.

[5] Tamura T. and Miyagi T., 1999, The effect of turbulence on aerodynamic forces on a square cylinder with various corner shapes, Journal of Wind Engineering and Industrial Aerodynamics, 83, 135145.

[6] Tamura T. and Miyagi T. Kitagishi T., 1998, Numerical prediction of unsteady pressures on a square cylinder with various corner shapes, Journal of Wind Engineering and Industrial Aerodynamics, 74, 531542.
[7] Kawai T., 1998, Effect of corner modification on aeroelastic instabilities of tall building, Journal of Wind Engineering and Industrial Aerodynamics, 74, 719- 729.

[8] Lakshminarayana, B., 1996, "Fluid Dynamics and Heat Transfer of Turbo-machinery”, Wiley \& Sons.

[9] Cengel, Y.A., and Cimbala, J.M., 2006, Fluid Mechanics: Fundamentals and Applications. Boston:McGraw-Hill Higher Education.

[10] Flow Modelling design Using CFD and Fluent software, 2001, Fluent Inc.

[11] Benson, G., 2009, Improving Fan System Performance, The New York Blower Company. 